\title{
Pathologic myopia: an overview of the current understanding and interventions
}

\author{
Takashi Ueta*, So Makino, Yuuka Yamamoto, Harumi Fukushima, Shigeko Yashiro, Miyuki Nagahara \\ Department of Ophthalmology, National Center for Global Health and Medicine, Tokyo, Japan
}

\begin{abstract}
Pathologic myopia is a major cause of low vision and blindness worldwide. Its social and economic burden has been demonstrated by epidemiological studies. There have been recent advances in the classification system for myopic maculopathy that enables clinicians to describe different types of lesions, including tessellated fundus, diffuse/patchy chorioretinal atrophy, macular atrophy, lacquer cracks, choroidal neovascularization (CNV), and Fuchs' spot, in a standardized format. From a therapeutic point of view, anti-vascular endothelial growth factor therapy has been established as first-line choice for myopic CNV. For myopic retinoschisis and macular holes with/without retinal detachment, pars plana vitrectomy has been generally accepted as an efficient strategy. Studies are being conducted to determine how to avoid the development of a postoperative macular hole and to improve the quality of vision after surgery. In recent years, studies have revealed preventive measures that can be taken against myopia progression, including low-dose atropine eyedrops and contact lens wearing with peripheral myopic defocusing.
\end{abstract}

Keywords: pathologic myopia, myopic maculopathy, myopic choroidal neovascularization, myopic retinoschisis

\section{Introduction}

Myopia has been a globally prevalent health issue and the incidence of myopia is expected to increase in the next few decades. Myopia is defined as refractive error by which the image coming into the eye is focused in front of, but not on, the retina. Both genetic and environmental factors are considered to play important roles in its development. In high myopic eyes, pathologic myopia, also known as "myopic macular degeneration", "myopic maculopathy", or "degenerative myopia" can occur, which can lead to visual acuity (VA) decline. Pathologic myopia affects $1-3 \%$ of the general population (1) and has been one of the major causes of low vision and blindness worldwide. In addition, because pathologic myopia often affects the productive age population, it is considered to be a social and economic burden.

In this review, current knowledge is summarized on the epidemiology of myopia, classification of myopic maculopathy, myopic choroidal neovascularization, myopic retinoschisis, and macular holes as well as interventions to suppress myopia progression.

\section{Epidemiology of myopia}

Epidemiology studies on myopia have been conducted in multiple locations around the world, and the reported prevalence and its impact on low vision and blindness have varied among the studies. Here are some examples.
A report from the Blue Mountains Eye Study that was conducted in Australia and published in 2002 investigated the prevalence of myopia and myopic retinopathy (2). In this urban population, aged 49 years or older, myopia was defined as $<-1.0 \mathrm{D}$ and was observed in $17 \%$ of the total participants. High myopia was defined as $<-5.0 \mathrm{D}$ and was observed in $2.7 \%$. In this study, myopic retinopathy was defined as a staphyloma, lacquer cracks, Fuchs' spot and myopic chorioretinal atrophy (CRA). Myopic retinopathy was observed in $1.2 \%$ of the population. The cases with myopic retinopathy were followed and significant progression was detected at re-examination after 5 years in $17 \%$ with an average deteriorated VA of nearly 2 logMAR lines (2).

In a report from the Rotterdam Study published in 1998, pathologic myopia was the third leading (6\%) cause of blindness and also the third leading (6\%) cause of low vision in subjects aged 55 years or older (3).

In a report from the Tajimi Study published in 2006, subjects aged 40 years or older were enrolled and high myopia of $<-5.0 \mathrm{D}$ was found in $8 \%$ of the participants, myopic macular degeneration was the third leading $(9.2 \%)$ cause of bilateral or monocular low vision and the first leading $(22.4 \%)$ cause of bilateral or monocular blindness (4).

In a report from the Beijing Eye Study published in 2006, degenerative myopia was responsible for the second leading $(32.7 \%)$ cause of low vision and also the second leading $(7.7 \%)$ cause of blindness. Cataract was 
the most frequent cause of both low vision and blindness. Myopia was the most frequent cause of both low vision and blindness in a population aged 40-49 years (5).

In the United States, the prevalence of myopia was $24 \%$ in the population aged 40 years and older (i.e., 34 million people), but it varied depending on ethnicity. The highest prevalence was for Caucasians (26\%), and lower for Hispanics (18\%), and African-Americans (15\%). Moreover, myopia prevalence is predicted to increase by 2050 such that there will likely be 45 million people aged 40 years and older affected (6).

In 2015, Rudnicka et al. reported results from a meta-analysis study that used quantitative Bayesian meta-regression on data from previous studies. The statistical model analyzed the prevalence of myopia (defined as $<-0.5 \mathrm{D}$ ) for different ages and ethnicities around the world, which constantly changes over time (7). The meta-analysis comprised 143 articles including over 350,000 subjects with more than 74,000 myopic cases. It disclosed the differences in myopia prevalence depending on ethnicity, age, environment, and gender. For example, myopia is most prevalent in East Asians, and at the age of 10 years old, the prevalence was already $35 \%$. At the age of 18 years old, the prevalence was as high as $80 \%$ in East Asians, while it was less prevalent in Caucasians (23\%) and black Africans (6\%). Children in an urban environment have 2.6-fold higher risk for developing myopia compared to those in a rural environment, and girls seem to have a 2-fold higher risk for developing myopia than boys, at least in Caucasians and East Asians (7).

While myopia itself is a major cause of vision loss, as described in these epidemiological studies, myopia also contributes to another vision-threatening disease; that is, glaucoma. Myopia is a risk factor for the development of glaucoma, and a previous meta-analysis has estimated that pooled odds for developing glaucoma is 1.65 and 2.46 for low and high myopic eyes, respectively (8). Therefore, detecting glaucoma in myopic eyes is important. This is often a diagnostic challenge for clinicians, however, due to myopic distortion and deformation in the optic nerve head and peri-papillary tissues (9). To diagnose myopic glaucoma, multimodal examinations through structural imaging and functional visual field tests are useful, however, care needs to be taken because they may increase the number of false diagnoses with glaucoma (9).

\section{Classification of myopic maculopathy}

High myopia is commonly defined as refractive error of $-5.0 \mathrm{D}$ or $-6.0 \mathrm{D}$ and/or axial length longer than 26.0 $\mathrm{mm}$ or $26.5 \mathrm{~mm}$. Pathologic myopia is highly myopic eyes with degenerative macular changes that present with different patterns and degrees of chorioretinal atrophy and specific findings including lacquer cracks, myopic CNV, and Fuchs' spot. Curtin et al. first reported the classification of myopic maculopathy in 1970 (10), and classifications have also been proposed by Avila et al. (11) and Hayashi et al. (12). However, international consensus on the classification of myopic maculopathy did not exist until a report published in 2015 described the classifications at an international consensus meeting (13). In this study, Ohno-Matsui et al. conducted a meta-analysis for pathologic myopia (META-PM) and proposed a new grading system for myopic maculopathy (Table 1, Figure 1) that was based on the long-term risk to develop myopic CNV and macular atrophy (14). Lacquer cracks, myopic CNV, and Fuchs' spot were defined as "plus" signs that are features that can lead to central vision loss.

\section{Myopic choroidal neovascularization (CNV)}

Myopic CNV is a serious complication in pathologic myopia that affects central vision (Figure 2). It recurs frequently and is usually bilateral. The long-term prognosis is poor, even if treated (15). The prevalence of myopic CNV is largely unknown, but a recent study based on the National Health and Nutrition Examination Survey and the American Academy of Ophthalmology's Intelligent Research in Sight (IRIS ${ }^{\circledR}$ ) Registry has estimated the prevalence among people aged 18 years and older in the United States as $0.017 \%$ (95\% CI, 0.0100.030) (16). The pathogenesis of myopic CNV is still largely unknown, but vascular endothelial growth factor (VEGF) levels in the aqueous humor are known to be elevated (15).

The funduscopic findings in myopic CNV can be staged as active, scarring, and atrophic, as classified by Tokoro in 1998 (17). Active myopic CNV is active neovessel formation that often occurs with a subretinal hemorrhage. For monitoring disease activity and decisions on re-treatment, VA, fluorescein angiography, and optical coherence tomography (OCT) are the vital examinations. Myopic CNV can lead to a scar formation with a hyperpigmented Fuchs' spot. In the long run, the macula becomes atrophic, forming CRA. Due to degenerative CRA progression in the atrophic stage, the eyes with myopic CNV undergo gradual VA decline over time even if the neovascular activity is successfully suppressed by treatment (15).

Regarding treatment for myopic CNV, intravitreal injections of anti-VEGF agents, including ranibizumab, aflibercept, or bevacizumab, have been the gold standard, which has been established through randomized controlled trials (18-20). The established regimen of anti-VEGF therapy is an initial injection followed by an additional injection when necessary, which is called the $1+$ pro re nata regimen (15). However, of note, the advantage gained through antiVEGF therapy is sustained for the initial several years, but it can gradually decline, and after 5-6 years, most of the VA gain might be lost (21). In the case that 
Table 1. Classification of myopic maculopathy based on international consensus meeting (13).

\begin{tabular}{ll}
\hline Category & Description \\
\hline $\begin{array}{l}\text { 1: Tessellated fundus } \\
\text { 2: Diffuse CRA }\end{array}$ & Well-defined choroidal vessels around the fovea and the arcade vessels. \\
3: Patchy CRA & Yellowish white appearance of posterior pole. \\
4: Macular atrophy & Well-defined, grayish white lesions in the macular area or around the optic disc. \\
"Plus" lesions & Well-defined, grayish white or whitish, round chorioretinal atrophic lesion in the foveal region. \\
Lacquer cracks & Breaks of the retinal pigment epithelium, Bruch's membrane, and choriocapillaris complex. \\
CNV & Exudation, hemorrhage, or serous retinal detachment at the posterior pole. \\
Fuchs' spot & Pigmented grayish white scar of myopic CNV without associated exudation.
\end{tabular}

The categories are in the order for long-term risk to develop myopic CNV and macular atrophy. "Plus" lesions are the additional features of myopic maculopathy that leads to visual loss regardless of the categorical stages.
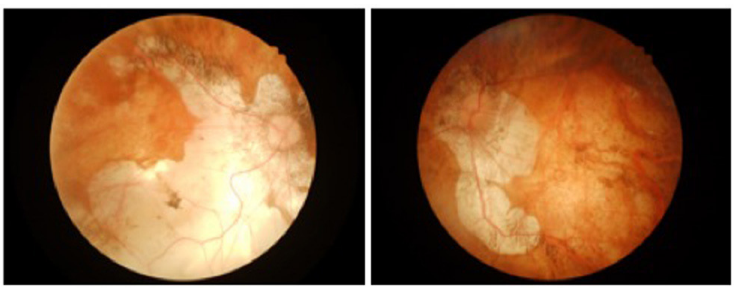

Figure 1. Fundus photographs of a patient with pathologic myopia. Both eyes are at the stage of macular atrophy (Category 4) associated with tessellated fundus, diffuse and patchy CRAs.

anti-VEGF therapy cannot be performed, verteporfin photodynamic therapy is an alternative choice of treatment, although the therapeutic effect is inferior.

\section{Myopic retinoschisis (MRS) and macular hole (MH)}

In high myopic eyes, typically with a posterior staphyloma, MRS can develop and lead to severe loss of VA. Retinoschisis is easily visualized on OCT, and MRS is observed in the outer retina, but it sometimes occurs both in the inner and outer retina. Regarding the pathogenesis of MRS, in addition to the posterior traction by a staphyloma, anterior traction by the epiretinal membrane (ERM) or adhesive vitreous cortex is considered to play an important role in the development of MRS (22). MRS is further associated with the development of a full-thickness $\mathrm{MH}$ with/without macular retinal detachment. These pathologies need to be treated surgically, and should not be performed late since long-standing MRS, even if successfully treated, does not result in sufficient postoperative visual recovery.

Standard surgical procedures used to treat MRS are pars plana vitrectomy with ERM removal plus inner limiting membrane (ILM) peeling. However, the postoperative course could be complicated by the development of a full-thickness $\mathrm{MH}$, for which several reports have recently suggested modifications of the surgical procedures. Described below is fovea-sparing ILM peeling and scleral imbrication.

The main purpose of fovea-sparing ILM peeling is to prevent the development of a postoperative fullthickness MH with or without retinal detachment, this surgical technique was first reported by Shimada et al. in

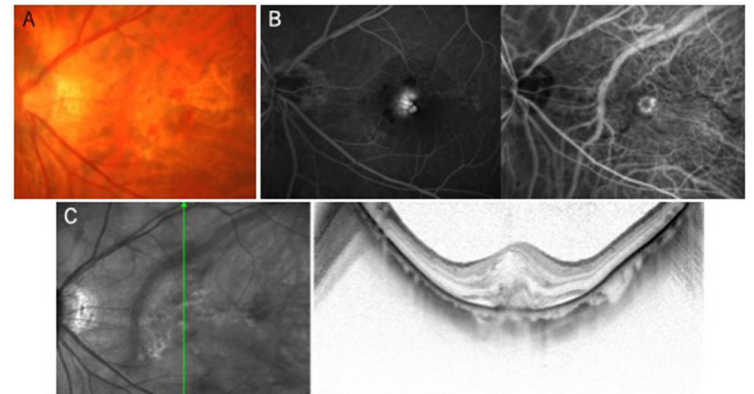

Figure 2. myopic CNV. (A) Color fundus photograph; (B) fluorescein (left) and indocyanine green (right) angiography; and (C) optical coherence tomography.

2012 (23). In this study, a full-thickness MH developed in 5 of 30 eyes $(16.7 \%)$ in which total ILM peeling was performed for MRS, whereas it developed in 0 of 15 eyes in which fovea-sparing ILM peeling was performed. In addition, postoperative VA was superior in eyes with fovea-sparing ILM peeling. Similar results have been reported in two other studies $(24,25)$, however, a recent study has argued that there was no difference in the postoperative VA between fovea-sparing and complete ILM peeling groups and that the rate of postoperative MH development was not significant $(2 / 20 v s .0 / 13 ; p=$ 0.508 ) (26). The potential benefit of fovea-sparing ILM peeling compared to complete ILM peeling has not been established yet and this needs to be addressed in the future.

The other modification in the surgical treatment for MRS is the use of scleral imbrication. Swan first reported this surgical technique for retinal detachment in 1959, and it has been applied to $\mathrm{MH}$ retinal detachment (27) and MRS (28). Baba et al. reported the application of scleral imbrication to MRS with PPV but without ILM peeling. They suggested that since postoperative full-thickness $\mathrm{MH}$ development is attributed to ILM peeling, scleral imbrication without ILM peeling can be an option other than fovea-sparing ILM peeling. The axial length reduction that scleral imbrication confers is not large (less than $1 \mathrm{~mm}$ after a year postoperatively), but the procedure could change the shape of the posterior globe and flatten the steep curvature of a staphyloma (28), which might help resolve MRS and prevent the development of a full- 


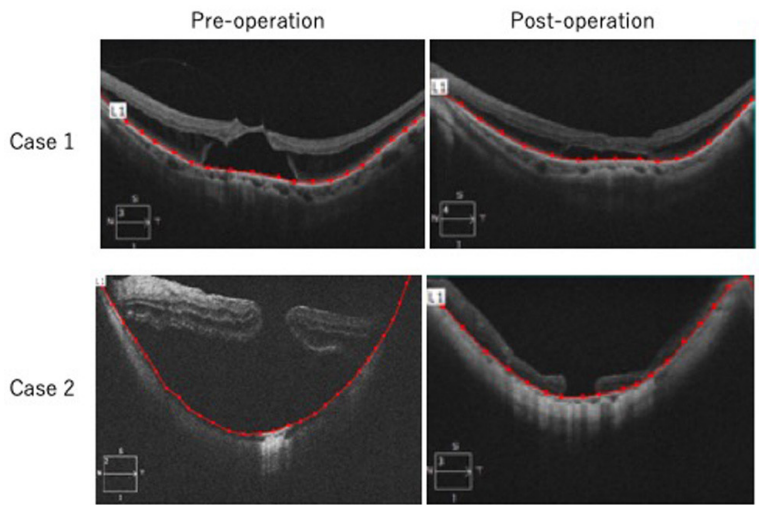

Figure 3. Flattened curvature of posterior fundus after lamellar scleral resection and infolding of the remaining sclera in patients with myopic retinoschisis and macular hole retinal detachment. Pre- and post-operative OCT in case 1 and 2 are shown. Posterior segment distance defined as the distance of retinal pigment epithelial line (red) using photo analyzer (AreaQ, Japan). Shorter posterior segment distance suggests flattened curvature of the posterior fundus after surgery.

thickness MH (Figure 3).

A drawback of scleral imbrication is that a significant postoperative astigmatism (about $4.0 \mathrm{D}$ worse at 1 month postoperatively and 2.0-3.0 D worse after 12 months compared to astigmatism before surgery) may develop $(27,28)$. Instead of scleral imbrication, our team uses scleral shortening through scleral invagination with lamellar scleral resection (29) (Figure 4), which usually causes less postoperative astigmatism.

\section{Suppression of myopia progression}

While medical and surgical therapies for pathologic myopia are important, the ultimate goal is suppression of myopia progression, which could lead to a significant reduction of eyes with pathologic myopia. Studies have revealed modifiable factors for the progression of myopia (30). For example, time spent outdoors, which is related to less dopamine release, is considered to suppress myopia progression. Indeed, low-dose antimuscarinic atropine eyedrops have been shown effective to suppress myopia (31). The other factor for myopia progression is peripheral hyperopic defocus. Not only recent studies have shown that orthokeratology (32) can reduce peripheral hyperopic defocusing and suppress myopia progression, but also a special contact lens that confers peripheral myopic defocusing has just been approved by Food and Drug Administration to be used for the suppression of myopia progression in children (33). However, long-term effectiveness and safety issues still remain (30).

\section{Conclusions}

In recent decades, remarkable advances have been made in understanding both the pathological basis and therapeutic strategies for pathologic myopia. Not
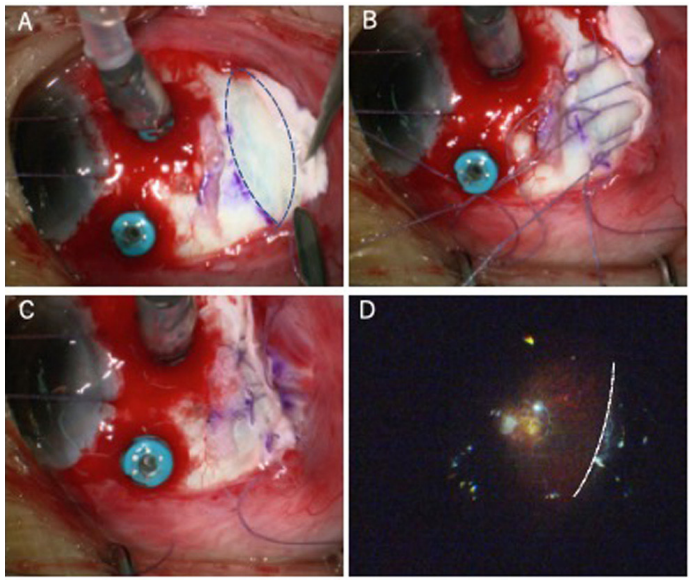

Figure 4. Intraoperative views of scleral shortening through scleral invagination with lamellar scleral resection to treat myopic retinoschisis. Dotted line in (A) indicates the area of lamellar scleral resection; (B) and (C) shows the infolding of the remaining sclera; dotted line in (D) indicates the infolded retina from inside of the eye under wide viewing system.

only are studies being performed to improve currently available therapies, but also novel therapies to suppress the progression of pathologic myopia are being considered for future studies.

\section{Acknowledgements}

This work was supported by the NCGM Intramural Research Fund (19A1020).

\section{References}

1. Wong TY, Ferreira A, Hughes R, Carter G, Mitchell P. Epidemiology and disease burden of pathologic myopia and myopic choroidal neovascularization: An evidencebased systematic review. Am J Ophthalmol. 2014;157:925.

2. Vongphanit J, Mitchell P, Wang J. Prevalence and progression of myopic retinopathy in an older population. Ophthalmology. 2002;109:704-711.

3. Klaver CC, Wolfs RC, Vingerling JR, Hofman A, de Jong PT. Age-specific prevalence and causes of blindness and visual impairment in an older population. The Rotterdam Study. Arch ophthalmol. 1998;116:653-658.

4. Iwase A, Araie M, Tomidokoro A, Yamamoto T, Shimizu $\mathrm{H}$, Kitazawa Y. Prevalence and causes of low vision and blindness in a Japanese adult population. The Tajimi Study. Ophthalmology. 2006;113:1354-1362.

5. Xu L, Wang Y, Li Y, Wang Y, Cui T, Li J, Li J, Jonas JB. Causes of Blindness and Visual Impairment in Urban and Rural Areas in Beijing. The Beijing Eye Study. Ophthalmology. 2006;113:1134.e1-11.

6. National Eye Insitute. Nearsightedness (Myopia) Data and Statistics. https://www.nei.nih.gov/learn-about-eyehealth/resources-for-health-educators/eye-health-dataand-statistics/nearsightedness-myopia-data-and-statistics (accessed January 12, 2020).

7. Rudnicka AR, Kapetanakis VV, Wathern AK, Logan NS, Gilmartin B, Whincup PH, Cook DG, Owen CG. Global variations and time trends in the prevalence of childhood 
myopia, a systematic review and quantitative metaanalysis: Implications for aetiology and early prevention. Br J Ophthalmol. 2016;100:882-890.

8. Marcus MW, De Vries MM, Junoy Montolio FG, Jansonius NM. Myopia as a risk factor for open-angle glaucoma: a systematic review and meta-analysis. Ophthalmology. 2011;118:1989-1994.e2.

9. Tan NYQ, Sng CCA, Jonas JB, Wong TY, Jansonius NM, Ang M. Glaucoma in myopia: diagnostic dilemmas. Br J Ophthalmol. 2019;103:1347-1355.

10. Curtin BJ, Karlin DB. Axial length measurements and fundus changes of the myopic eye. Trans Am Ophthalmol Soc. 1970;68:312-334.

11. Avila MP, Weiter JJ, Jalkh AE, Trempe CL, Pruett RC, Schepens CL. Natural history of choroidal neovascularization in degenerative myopia. Ophthalmology. 1984:91:1573-1581.

12. Hayashi K, Ohno-Matsui K, Shimada N, Moriyama M, Kojima A, Hayashi W, Yasuzumi K, Nagaoka N, Saka N, Yoshida T, Tokoro T, Mochizuki M. Long-term pattern of progression of myopic maculopathy: a natural history study. Ophthalmology. 2010;117:1595-1611.e4.

13. Ohno-Matsui K, Kawasaki R, Jonas JB, et al. International photographic classification and grading system for myopic maculopathy. Am J Ophthalmol. 2015;159:877-883.e7.

14. Fang Y, Yokoi T, Nagaoka N, Shinohara K, Onishi Y, Ishida T, Yoshida T, Xu X, Jonas JB, Ohno-Matsui K. Progression of Myopic Maculopathy during 18-Year Follow-up. Ophthalmology. 2018;125:863-877.

15. Cheung CMG, Arnold JJ, Holz FG, Park KH, Lai TYY, Larsen M, Mitchell P, Ohno-Matsui K, Chen SJ, Wolf S, Wong TY. Myopic Choroidal Neovascularization: Review, Guidance, and Consensus Statement on Management. Ophthalmology. 2017;124:1690-1711.

16. Willis JR, Vitale S, Morse L, Parke DW, Rich WL, Lum F, Cantrell RA. The Prevalence of Myopic Choroidal Neovascularization in the United States: Analysis of the IRIS ${ }^{\circledR}$ Data Registry and NHANES. Ophthalmology. 2016;123:1771-1782.

17. Tokoro T. Types of fundus changes in the posterior pole. In: Atlas of posterior fundus changes in pathologic myopia. Springer, Tokyo, 1998; pp. 5-22.

18. Hayashi K, Ohno-Matsui K, Teramukai S, Shimada N, Moriyama M, Hayashi W, Yoshida T, Tokoro T, Mochizuki M. Comparison of visual outcome and regression pattern of myopic choroidal neovascularization after intravitreal bevacizumab or after photodynamic therapy. Am J Ophthalmol. 2009;148:396-408.e1

19. Wolf S, Balciuniene VJ, Laganovska G, Menchini U, Ohno-matsui K, Sharma T, Wong TY, Silva R, Pilz S, Gekkieva M; RADIANCE Study Group. RADIANCE : a randomized controlled study of ranibizumab in patients with choroidal neovascularization secondary to pathologic myopia. Ophthalmology. 2014;121:682-692.

20. Ikuno Y, Ohno-Matsui K, Wong TY, Korobelnik JF, Vitti R, Li T, Stemper B, Asmus F, Zeitz O, Ishibashi T; MYRROR Investigators. Intravitreal aflibercept injection in patients with myopic choroidal neovascularization: the MYRROR study. Ophthalmology. 2015;122:1220-1227.

21. Ruiz-moreno JM, Montero JA, Araiz J, Arias L, Garcíalayana A, Carneiro A, Figueroa MS, Silva R. Intravitreal anti-vascular endothelial growth factor therapy for choroidal neovascularization secondary to pathologic myopia: Six years outcome. Retina. 2015;35;2450-2456.

22. Shinohara K, Tanaka N, Jonas JB, Shimada N, Moriyama
M, Yoshida T, Ohno-Matsui K. Ultrawide-field OCT to investigate relationships between myopic macular retinoschisis and posterior staphyloma. Ophthalmology. 2018;125:1575-1586.

23. Shimada N, Sugamoto Y, Ogawa M, Takase H, OhnoMatsui K. Fovea-sparing internal limiting membrane peeling for myopic traction maculopathy. Am J Ophthalmol. 2012;154:693-701.

24. Iwasaki M, Miyamoto H, Okushiba U, Imaizumi H. Fovea-sparing internal limiting membrane peeling versus complete internal limiting membrane peeling for myopic traction maculopathy. Jpn J Ophthalmol. 2020;64:13-21.

25. Tian $\mathrm{T}$, Jin $\mathrm{H}$, Zhang Q, Zhang $\mathrm{X}$, Zhang H, Zhao P. Long-term surgical outcomes of multiple parfoveolar curvilinear internal limiting membrane peeling for myopic foveoschisis. Eye. 2018;32:1783-1789.

26. Wang L, Wang Y, Li Y, Yan Z, Li Y, Lu L, Lu T, Wang $X$, Zhang S, Shang Y. Comparison of effectiveness between complete internal limiting membrane peeling and internal limiting membrane peeling with preservation of the central fovea in combination with $25 \mathrm{G}$ vitrectomy for the treatment of high myopic foveoschisis. Medicine (Baltimore). 2019;98:e14710.

27. Fujikawa M, Kawamura H, Kakinoki M, Sawada O, Sawada T, Saishin Y, et al. Scleral imbrication combined with vitrectomy and gas tamponade for refractory macular hole retinal detachment associated with high myopia. Retina. 2014;34:2451-2457.

28. Baba T, Tanaka S, Oshitari T, Yamamoto S. Scleral imbrication combined with pars plana vitrectomy without internal limiting membrane peeling for myopic. Retina. 2016;36:1927-1934.

29. Everett W. A new scleral shortening operation: preliminary report. AMA Arch Ophthalmol. 1955;53:865869.

30. Morgan IG, French AN, Ashby RS, Guo X, Ding X, He M, Rose KA. The epidemics of myopia: aetiology and prevention. Prog Retin Eye Res. 2018;62:134-149.

31. Chia A, Lu QS, Tan D. Five-year clinical trial on atropine for the treatment of myopia 2 myopia control with atropine $0.01 \%$ eyedrops. Ophthalmology. 2016;123:391399.

32. Swarbrick HA, Alharbi A, Watt K, Lum E, Kang P. Myopia control during orthokeratology lens wear in children using a novel study design. Ophthalmology. 2015;122:620-630.

33. Food and Drug Administration. FDA approves first contact lens indicated to slow the progression of nearsightedness in children. https://www.fda.gov/news-events/pressannouncements/fda-approves-first-contact-lens-indicatedslow-progression-nearsightedness-children (accessed January 14, 2020).

Received February 2, 2020; Revised May 5, 2020; Accepted May 8, 2020

Released online in J-STAGE as advance publication May 11, 2020.

*Address correspondence to:

Takashi Ueta, Department of Ophthalmology, National Center for Global Health and Medicine, 1-21-1 Toyama Shinjuku-ku, Tokyo 162-8655, Japan.

E-mail: ueta-tky@umin.ac.jp 